\title{
Classroom Translation: The Case of Iranian University Students
}

\author{
Karim Sadeghi \\ Islamic Azad University, Salmas Branch, Iran \\ Email: k.sadeghi@urmia.ac.ir
}

\begin{abstract}
Translation research has been of interest to both practitioners and theoreticians in the field. As far as the practical side of the coin is concerned, translation problems have been studied with an eye to the role of inter-lingual and intra-lingual transfer. The research reported here was an attempt to understand the nature of translation problems of Iranian EFL learners. For this purpose, three cohorts of EFL learners taking introductory and advanced translation courses (from English to Persian and vice versa) participated in the study in order to identify the sources of their translation problems and offer solutions for the betterment of the situation. The content analysis of the samples of translation activities of the participants reveals that translation is a more sophisticated skill than expected and that an inappropriate linguistic competence in English is responsible for the bulk of translations blunders made by learner translators.
\end{abstract}

Index Terms - translation, interpretation, Iranian context, EFL learner

\section{INTRODUCTION}

One of the skills EFL learners are expected to master by the end of their university education is the ability to communicate in English to the desired level. One aspect of this communicative ability is the mastery learners gain in translating either from English to Persian or vice versa. While there are so many courses offered for EFL majors in translation (including The Principles of Translation, Translating Simple Texts, and Advanced Translation), the output of such translation courses is usually less than satisfactory. My experience as a translation instructor in Salmas Azad University for the last 4 years has shown me that even after getting pass marks in translation courses, the majority of the students fail to transfer this skill to their future real-life practices. Different students face different sorts of translation problems which could be attributed to a variety of reasons including the lack of sufficient language base in terms of vocabulary and grammar, problems in comprehension which is I suppose a pre-requisite for translation and the like.

As such, it was the aim of this research to look at the translation practices of a cohort of EFL majors studying ELT and English Translation in Salmas Azad University in an attempt to discover first of all the patterns of problems most frequently encountered by such learners, secondly to discover the underlying causes of such problems, and finally to suggest solutions for the betterment of the condition.

\section{REVIEW OF RELATED LITERATURE}

It can be claimed that translation has existed since human beings came into the world. People with different languages had to make contacts with each other out of necessity, and while some messages could be communicated via sign language and using onomatopoeic words common to all languages, for the success of proper communication, translation was inevitable. Indeed translation became known as a science on its own in mid-20 $0^{\text {th }}$ century when structural linguists promoted the place of contrastive analysis in studying foreign languages and the term was highlighted by the choice of the words for the title of Nida' (1964) book: Toward a science of translation.

Much has been said and written about the meaning and importance of translation. There are hundreds of books and more so research papers on different aspects of translation across the world. The term 'translation' has been defined slightly differently by different scholars. Translation is defined by Richards and Schmidt (2002, p. 563) as 'the process of rendering written language that was produced in one language (the SOURCE LANGUAGE) into another language (the TARGET LANGUAGE), or the target language version that results from the process.'

Most other definitions of translation, although a bit different in wording, have kept more or less the same concept. For example, Munday (2001, p. 5) has the following to say about translation process (cited in Mousavei Mianagh and Mohammadi Dehcheshmeh, 2009, p. 13):

The process of translation between two different written languages involves the translator changing an original written text (the source text or ST) in the original verbal language (the source language or SL) into a written text (target text or TT) in a different verbal language (the target language or TL).

Different theories of translation have also emerged (such as product-oriented descriptive translation theory, functionoriented descriptive translation, process-oriented descriptive translation, medium restricted translation theory, arearestricted theory and so on) in which translation have been looked at and defined from a bit different perspectives. 
However, as our focus is more practical rather than theoretical, we do not deem it relevant to delve into such theories and we limit ourselves to issues of a more practical nature and those immediately usable by classroom teachers and learners of translation.

There are many commonalities, of course, between the definitions provided for the term. For example, if we compare the two most-commonly cited definitions by Nida (1964) and Catford (1965), we can easily understand that in both definitions, translation is viewed as an activity of changing a piece of text from one language into another. Nida (1964) defines translation as the exercise of producing a text in the target language as closely equivalent as possible to the original text in the source language in the form, the style and the meaning. In a much similar vein, Catford (1965) equates translation with the replacement of textual material in one language by equivalent textual material in another language. The striking feature of these two views on the meaning of translation is that the reproduced text (as the evidence of translation) should be equivalent to the original one. In order to arrive at this equivalence, therefore, the translator should first be able to comprehend the original passage in the intended line (taking into consideration the particular contextual circumstances under which it was produced) and make a conscious attempt to transfer all this to another language such that the translated text will have the same locutionary meaning, illocutionary force and perlocutionary effect on its reader (in translated form) as the original text will have on its reader (in its intact form).

Research on translation has a relatively long tradition. Formal research on translation began when translation started to be known as a science in mid $20^{\text {th }}$ century. Research on translation has focused on both theoretical and practical issues. Since our concern is with classroom applications of translation, we will limit ourselves to a sample of these latter types of research publications.

Liao (2006) claims that language learners use translation as a strategy to comprehend, remember and produce a foreign language. Liao's research focused on the use of translation among 351 Taiwanese college students and their views on using the strategy. The data gathered through questionnaires and an interview revealed that participants felt that translating helped them acquire English language skills such as reading, writing, speaking, vocabulary, idioms, and phrases.

The role of translation in L2 vocabulary learning was investigated by Mummel (2010). Her study focused on 191 French speakers who were enrolled in an introductory linguistics course in English as a second language who received treatment under three conditions: L1 to L2 active translation $(n=71)$, L2 to L1 active translation $(n=71)$, and exposure and copy exercise $(n=49)$. The findings suggested that active translation from both L1 into L2 and vice versa benefitted the learners, while as far as short-term recall of L2 vocabulary was concerned, the students were 'likely to benefit more from exposure to new vocabulary accompanied by translation equivalents in a copy exercise' (p. 71).

As far as translation problems of language learners in the Iranian context are concerned, the following recent studies are worth mentioning. Anani Sarab and Eshraq (2010) studied the problems of Iranian translators in the translation of proper nouns in young adults' imaginative literature focusing on two book series (Trilogy of Inheritance and Deltora Quest). They found that Persian translators usually adopt the easiest procedure for translating proper nouns from English into Persian by using the strategy of transcription rather than a semantically-loaded translation where the focus could be on connotative meaning. The translators were also reported to have used other translating techniques such as substitution in which they invented a name for foreign sources and used a neologism. They also used couplet strategy for translating proper names in which they selected a Persian name which bore some of the referent's characteristics.

Investigating gender differences in translating metaphors and trying to find an answer to the question of whether there is any relationship between translator's gender and strategies employed in translating metaphors, Farahzad and Faridzadeh (2009) administered a test of translation made of 24 contextualized metaphors to 32 female and 23 male MA students of Translation studying at Allameh Tabatabie University, Azad University (Science and Research, and Arak Branches) after homogenizing them using an adapted version of TOEFL IBT. They came out with the following results: the most frequent strategy used by both groups was reducing metaphor to its sense and the least frequently used strategy was keeping the metaphor and providing the sense at the same time; the second frequent strategy used by both groups was to keep metaphor, with the third being the omission of the metaphor. Both males and females followed exactly the same order of frequency of strategy use; male translators retained the metaphors more than females; female translators showed more tendencies toward explaining the meaning of metaphors and used more similes.

Interested in identifying general strategies that learner translators use while doing translation activities, Shirvani and Ranjbar (2009) conducted a qualitative study with eight female undergraduate students doing Translation at Azad University (Gha'emshahr Branch). The researchers used a think-aloud procedure for data collection purposes after training the participants during four preparatory sessions before the final experiment began. The analysis of the thinkaloud protocols revealed several types of strategies used by trainee translators: Cognitive strategies (including inferencing, search strategies, equivalent retrieval, and reduction); Meta-cognitive strategies (including evaluation decision-making and self-monitoring); social strategies (such as brainstorming and correction); and finally affective strategies.

\section{METHOD}

\section{A. Participants}


The participants of this research project were 25 sophomore (in groups of 12 and 15) and 17 junior students majoring in ELT and English Translation at Salmas Azad University. They were taking translation courses 1 and 2 respectively during the Spring semester of 2010. The sample was an opportunistic one for the reasons of logistics (Mackey and Gass, 2005). The age range of the participants ranged from 19 to 31 years and three quarters of the participants were female learners. All the participants spoke Farsi (as L2) and English as L3. Azeri was the first language of 80 per cent of them with the rest having Kurdish as L1.

\section{B. Instruments}

The major materials used in the study were excerpts of passages used in the classrooms every session for practice purposes. Various passages at appropriate levels were chosen from newspapers and magazines (both English and Persian) and were given to learners to be translated every classroom session. Each session, usually one English and one Farsi passage was allocated for translation.

\section{Procedure}

The classroom translations of at least one English text into Farsi and vice versa were regular classroom practices during translation classes the researcher had taught. Every learner was required to have a note book for this purpose from the beginning of the semester. They were asked to select two texts at their desired level and interest area from any source (and preferably from newspapers and magazines) and cut and paste them into their notebooks and write their own translations on the opposite page. So different students selected texts of different types, of different disciplines and at more or less similar difficulty levels as the texts tended to correspond to their pre-intermediate and intermediate proficiency level.

Every session, at the start of the class, the researcher checked the assignments of all students and selected two short texts based on the level of the class or certain translation issues which needed focusing on and further discussion. The texts were written on the board and depending on the difficulty level of the text and its length, a certain amount of time was allocated for each student to work independently on its translation. Then one of the translations done by the students (usually one from which some teaching and learning can benefit the students) was written on the board. Each student produced his own version of translation for the text on the board and these translations were collected at the end of each session or the semester for later analysis by the researcher.

\section{FINDINGS AND DISCUSSION}

The study included translation samples from 3 groups of EFL learners taking Translation 1 and Translation 2 courses at Islamic Azad University of Salmas as mentioned above. In the following analysis, a sample original sentence offered for translation (both Persian and English) will be provided with examples of translations produced by learners in Groups $\mathrm{A}, \mathrm{B}$, and $\mathrm{C}$ respectively. The quantity of the wrong translations will be indicated and the possible sources of errors will be identified following the model provided by Sadeghi (2009). To make the analysis and discussion more meaningful and more appropriate for English readership, the Farsi texts (whether source texts or translated versions of English texts) have been back-translated into English and indicated as such.

Group A was made of 12 sophomores taking Translation 1 course (Translating Simple Texts). Except for 2 students, all the rest were female. The age range of the participants in neither group was checked as it was not felt to be an important variable, since this study was mainly qualitative in which age or gender had to play little role as moderators. However, the participants here were more or less homogeneous in their early 20's. The group members were requested to translate 7 English mini-texts into Persian and 7 Persian texts into English. The following is an account of a translation of the group for a sample English and a sample Persian mini-text.

English source text: Russia has hailed the new US administration's intention to start talks soon on a successor deal to the 1991 Strategic Arms Reduction Treaty, which expires in December.

The text produced substantial problems for translators. One reason behind learners' problematic translations in this and other similar cases below may be that the texts were political in nature about which students probably did not have much background and schemata, as the students has been asked to cut texts from newspapers (although they were free to choose any text related to any filed). As such, although the major excuse behind most translation problems remains to be the translator's linguistics competence or systemic knowledge (Widdowson, 2007), the role of conceptual information or schematic knowledge (Widdowson, 2007) cannot be neglected. The above text was translated by only 10 students none of whom produced an acceptable version which was equivalent to the source in the loosest sense of the word.

Amongst the most noticeable blunders observed in the translation of the above text are the following. The use of 'attempts' instead of 'intention' by a majority of translators; the use of 'new intentions' and 'new administration procedure' instead of 'new US administration'; the use of 'pre-mature talks' instead of 'start talks soon'; the use of 'the Treaty that expired in 1991' instead of 'the 1991 strategic Arms Reduction Treaty'; the omission of 'in December' in most translations; the use of 'new US administration's speech' instead of 'new US administration's intention to start talks', the use of 'facilities' instead of 'Arms' (the spelling of words being very close in Farsi: 'tashilat' vs. 'taslihat'); and the use of 'talks with a successor' instead of 'talks on a successor deal'. Apart from these problems the sources of which can be 
traced to both linguistics and non-linguistics issues as discussed above, there were incomplete translations and those following English sentence patterns.

Persian source text (back-translated into English): According to Arya, in interview with CBS TV Channel, Obama claimed that Iran's international isolation is increasing and that America seeks to get help from a united international community to confront the country's nuclear program.

Except for one student, the rest tried their hand in producing a translation for the above sentence in Persian. Neglecting minor grammar and style problems in one translation which made it more or less acceptable, none of the other translations could really be called translations, with two of them left incomplete. The biggest shock is found at the beginning of most translations: much to the researcher's surprise and disappointment, 'Obama' had been taken literally by many translators and translated as 'he to we', 'he with us', and 'Obama with us'. One wonders whether this mistake can at all be tolerated. Certainly, the candidates at this age and social status are expected to have known who Obama was, however little their political and geographical world knowledge is expected to be. If such mistakes can be made by a good number of university students, the rest of story can easily be understood. A similar scenario was also seen in the performance of one student: Instead of 'According to Arya' which is the back-translated version of 'Arya nevesht' in Persian, the student has taken the phrase at face value, regarding it as a proper noun and has used the same in English as translation equivalent; that is, instead of 'According to Arya', she had produced 'Aryanevesht'.

In addition to these obvious goofs which are like jokes rather than actually produced data, examples of more serious problems were as follows: the use of 'claimant' instead of 'claimed', the use of 'global seclusion' instead of 'international isolation', the use of 'for comparison', 'opposite with', 'for contrast with' and 'for confront with' instead of 'to confront' which was the back-translated form of 'baraye mogabekle ba'; the use of 'by pursuing to help', 'after get help', 'pursuing given help', 'looking for get help', 'pursuing given helped' and 'try to give help' instead of 'seeking for help' which is the back-translated form of 'dar paye komak gereftane az'; and finally, the use of 'internal society united', 'international community united' and 'international society united' instead of 'united international community'. Although the trace of L1/L2 interference is evident in the cases where a preposition is involved such as in 'for confront with', the major source of the problem as always seems to be the translators' linguistics competence in the target language since with enough command of the language, those L1/L2-related episodes could easily be prevented. Such negative interference is a characteristic of beginner learners, but the candidates in question can no longer be viewed as beginners, although their performance does not allow them to be labeled intermediate either.

Group B was made of 15 sophomores taking Translation 1 course (Translating Simple Texts). Except for 2 students, all the rest were females. The group members were requested to translate 6 English mini-texts into Persian and 7 Persian texts into English.

English source text: Ankara (PPA): Thousands of people gathered in Ankara yesterday in support of a number of academics who were charged on Friday of being members of the so-called Ergenekon Conspiracy to overthrow Turkey's moderate Islamic government.

Apart from 3 candidates who did not produce a translation and 2 whose translations were left incomplete, the rest produced a more or less acceptable translations semantically speaking. Except for one student, no others seemed to have seen the beginning part of the text (i.e. Ankara (PPA)) or neglected it on the assumption that it did not need to surface in the translation. Although most translations were acceptable in content, word-order and minor style problems were detected in a good number of them. The word 'academics' was for exampled translated into 'university students', 'university professors' and 'student unions' in a few cases. One candidate had also misunderstood the meaning of 'were charged' equating it with a similar expression 'were in charge of', which can be indicative of both a lack of attention or even a linguistic deficiency in distinguishing two meanings of charge. One student also seemed to have a problem with the final part of the sentence, translating it to '... whose aim was to destroy the Islamic government's tranquility'. The problem seems to have arisen from the inability of the candidate to assign the intended meaning to the word 'moderate' which means 'middle-of-the-road' in the above text rather than 'tranquility' as wrongly assumed by the trainee translator.

Persian source text (back-translated into English): Vegetable juice is among the drinks the daily use of which not only transfers the nutrients in the vegetables to the body but also causes a weight loss.

Almost every candidate attempted a translation, but almost all were faulty in a way or another. Among the most noticeable problems were the use of 'vegetable water' or 'water of vegetables' instead of 'vegetable juice', indicating a word for word translation activity by many novice translators. The construction 'the daily use of which' or 'whose daily use' was translated in a structurally inappropriate way by almost all candidates to such constructions as 'whose daily use it', 'that daily use of them', 'the daily use of that', 'that its daily use', 'whose the daily use of that', 'that its daily use', 'which daily using', 'that it's daily using', 'that it is daily used', 'which daily using them' and so on, representing a clear and big gap in the learners' knowledge of English relative clauses beginning with 'whose' or including 'of which'.

The candidates' weak English proficiency also tempted them to use circumlocution and explain the meaning in a few cases rather than trying to stick to the pertinent and succinct English structure. For example, instead of using 'causes weight loss', they used constructions, ungrammatical at times, such as 'become cause of reduce of weight', 'cause lose of weight', 'cause loss of weight', 'cause weight of body to decrease', 'reduce the weight of body', 'cause loosing weight', 'make low the weight' and the like. There were also instances of improper choice of vocabulary equivalents such us using 'food materials' and 'articles of food' instead of 'nutrients', 'reach' instead of 'transfer', 'but only' instead of 'but also', 
all of which except for the final replacement which may be the result of a fatigue or a performance issue are justified by the fact that the learners' L2 competence was not well developed at the time to make fine distinctions between vocabulary items.

Group $\mathrm{C}$ consisted of 17 juniors taking Translation 2 course (Advanced Translation). All the class members were females except for 2 of them. The group members were requested to translate 5 English mini-texts into Persian and 6 Persian texts into English.

English source text: Significant progress in realizing women's rights is crucial to achieving sustainable development and the globally agreed targets to slash poverty, hunger, illiteracy and a host of other socio-economic illnesses, the Deputy Security General said on Thursday.

Except for one candidate with no translation and two with partial translations, the rest of the students provided a sort of more or less acceptable translation, generally speaking. The two most noticeable areas of problem were in omitting 'a host of' from translations and inappropriate translations of 'the globally agreed targets' into back-translated structures like 'global agreement in targets', 'agreeing with global targets' and 'agreed global targets', all of which indicate a weakness in appropriate mastery of the complex target structure in English when an adverb precedes a adjective. Less important obstacles were also observed similar to the cases reported above, for example in using less appropriate vocabulary equivalents at times, sentence structure order, punctuations and so on, but these common problems are not taken care of any further as they do not add new categories to the sorts of issues we came across.

Persian source text (back-translated into English): It should be said with great sorrow that there are many examples of this kind in our administrative society; for example, in Cultural Department, whose unique position and sensitivity are continuously highlighted, a manager is appointed who neither enjoys any work experience in the field, nor does his education have any relationship with his job; and it is clear what will happen to the culture of such a country.

Apart from a no-translation case and a few incomplete cases, most translations enjoyed a good level of acceptability. They were legible but with numerous minor structural and vocabulary problems, with the overall structure making sense. As the initial structure of the sentence does not have any subject in Persian, that problem did show itself in a good number of cases: 'should said sorrowfully that', 'With great sorrow should tell that', and 'unfortunately we should be said that'. Although a zero structure in the source language may be argued to blame for the mistake, lower L2 proficiency is the ultimate cause as with a greater mastery of L2 structure, the candidates could have avoided such funny subject-less structures. Incorrect word choices were as usual highlighted such as the use of 'culture part' and 'culture section' instead of 'Cultural Department' or the use of 'many patterns' and 'many samples' instead of 'many examples' for the Persian phrase 'nomuneh-haye ziadi'. The use of 'job background' and 'working background' instead of 'work experiences' was another similar scenario.

\section{CONCLUSION}

This research study was set up with the intent of analyzing the translations problems Iranian EFL learners faced in doing simple translations. Cohorts of sophomore and junior EFL majors at Islamic Azad University of Salmas received translation training and practice for the period of a university semester. Excerpts of their homework were selected for further focus and analysis. Both English-Persian and Persian-English texts were considered. The ultimate finding after a lengthy content and discourse analysis a sample of which was reported above indicated that most learner translators face serious translations problems as soon as their immediate linguistics repertoire is challenged. The candidates faced the most problems in Persian-English translations since it was much easier to comprehend than to compose English. Generally speaking, most of the problems dealt with word-order and structural points as well as inappropriate vocabulary choice, which in most cases distorted the message completely, rendering something much different from what was already meant. The biggest share of the problems documented in this study is connected to a low L2 proficiency, although the role of L1 transfer was evident especially in the cases where a collocational or prepositional structure was involved.

Although this study was based on small sample of EFL candidates learning translation in a small university and may thus be regarded as a case, the findings of which are not generalizable, it is our belief that since the major variable affecting translation success is the candidates' proficiency in the target language (English here), similar findings are expected in other similar contexts where the participants have similar language proficiency backgrounds. That is why despite the fact that we fail from extending our findings to larger populations, we remain confident that with samples taken from other contexts, not very dissimilar patterns of problems are expected to be reported. We are also aware that certain other variables such as the translators' L1, the time available for the translation activity, the amount of help provided during the activity, the existence of an exam as opposed to a non-exam setting, the text topic, the familiarity of the candidates with the content as well as many other issues will play their role in the final outcome. That is why the best conclusion and generalization we can offer for the time being is that our findings are tentative and open to replication by fellow researchers.

\section{REFERENCES}

[1] Anani Sarab, M.R., \& Eshraq, B. (2010). Translation of proper nouns: The case of young adults' novels translated from English 
into Persian. Translation Studies, 7(28): 9-30.

[2] Catford, J. C. (1965). A linguistics theory of translation: An essay in applied linguistics. London: Oxford University Press.

[3] Farahzad, F., \& Faridzadeh, F. (2009). Gender and translation metaphors. Translation Studies, 7(27): 81-96.

[4] Hummel, K.M. (2010). Translation and short-term L2 vocabulary retention: Hindrance or help? Language Teaching Research, 14(1): 61-74.

[5] Liao, P. (2006). EFL learners' beliefs about and strategy use of Translation in English learning. RELC, 37(2): 191-215.

[6] Mackey, A., \& Gass, M.S. (2005). Second language research: Methodology and design. NJ: Lawrence Erlbaum Associates.

[7] Miemadi, S.A. (1991, 1997). Theories of translation and interpretation. Tehran: SAMT.

[8] Mousavi Mianagh, T., \& Mohammadi Dehcheshmeh, M. (2009). Translation studies. Tehran: Noavarne Sharif.

[9] Nida, E. (1964). Towards a science of translation with special reference to principles and procedures involved in Bible translating. Leiden: Brill.

[10] Richards, J.C., \& Schmidt, R. (2002). Longman dictionary of language teaching and applied linguistics (3rd ed.). London: Longman.

[11] Sadeghi, K. (2009). Translation, interpretation, or composition: The art of transition. Translation Studies, 7(27): 65-80.

[12] Shirvani, K., \& Ranjbar, S. (2009). Identifying translator trainees' strategies: A think-aloud protocol analysis. Translation Studies, 7(27): 97-110

[13] Widdowson, G.H. (2007). Discourse analysis. Oxford: OUP.

Karim Sadeghi is an assistant professor of TESOL and Head of English Language Department at Urmia University. His contributions have appeared in The Modern Language Journal, Canadian Journal of Linguistics, TESL Canada Journal, Asian EFL Journal, The Asia Pacific Education Researcher among others. 